\title{
Search for Radio Recombination Lines towards the Gravitational Lens PKS1830-211
}

\author{
N.R. Mohan ${ }^{1,2}$, K.R. Anantharamaiah ${ }^{1}$ and W.M. Goss ${ }^{3}$ \\ 1 Raman Research Institute, Bangalore 560080, India, \\ 2 Indian Institute of Science, Bangalore 560012, India \\ 3 NRAO, Socorro, NM 87801, USA
}

\begin{abstract}
A search for radio recombination lines near $20 \mathrm{~cm}$ at $\mathrm{z}=0.193$ and $\mathrm{z}=0.886$ towards the gravitational lens system PKS1830-211 has yielded upper limits of $\left|\tau_{L}\right| \leq 5 \times 10^{-5}$ and $\leq 5 \times 10^{-4}$ at the two redshifts respectively. Based on the non-detections, we derive upper limits to the emission measure of the ionized gas in the absorbing systems. We also present continuum flux density measurements over the frequency range $0.3-45 \mathrm{GHz}$ made at a single epoch.
\end{abstract}

\section{Introduction}

There is evidence that the gravitational lens system PKS1830-211 has two absorbers at two different redshifts of 0.886 and 0.193 . The main lens, a normal galaxy at $\mathrm{z}=0.886$, has been studied in $\mathrm{HI}, \mathrm{OH}$ and a host of molecular lines (Chengalur, de Bruyn, \& Narasimha 1999 and references therein). The only evidence for an additional absorber at $\mathrm{z}=0.193$ is from $\mathrm{HI}$ absorption (Lovell et al. 1996; Verheijen et al. 1999) studies. Searches for molecular lines at this redshift have been unsuccessful.

\section{Observations}

Taking advantage of the strong radio continuum of the background source $\left(\mathrm{S}_{1.4 \mathrm{GHz}}=10 \mathrm{Jy}\right)$, we searched for stimulated emission recombination lines at both redshifts, using the $\mathrm{VLA}^{1}$ at $20 \mathrm{~cm}$; the $\mathrm{H} 158 \alpha$ line from the $\mathrm{z}=0.193$ system $\left(\nu_{\text {rest }}=1.65 \mathrm{GHz}\right)$ using a bandwidth of $1.56 \mathrm{MHz}$ and a velocity resolution of $5.3 \mathrm{~km} \mathrm{~s}^{-1}$ and the $\mathrm{H} 136 \alpha$ line from the $\mathrm{z}=0.886$ system $\left(\nu_{\text {rest }}=2.59 \mathrm{GHz}\right)$ using a bandwidth of $3.125 \mathrm{MHz}$ and a resolution of $21 \mathrm{~km} \mathrm{~s}^{-1}$. Neither line was detected with $5 \sigma$ upper limits to $\left|\tau_{L}\right|$ of $5 \times 10^{-5}$ and $5 \times 10^{-4}$ corresponding to $\mathrm{z}=0.193$ and $\mathrm{z}=0.886$ respectively.

Since this source is highly variable, we used the VLA to measure the continuum flux density at a single epoch over the frequency range $327 \mathrm{MHz}$ to $45 \mathrm{GHz}$, to determine the intrinsic spectrum of the background source. The observations

\footnotetext{
${ }^{1}$ The National Radio Astronomy Observatory is a facility of the National Science Foundation operated under cooperative agreement by Associated Universities, Inc.
} 

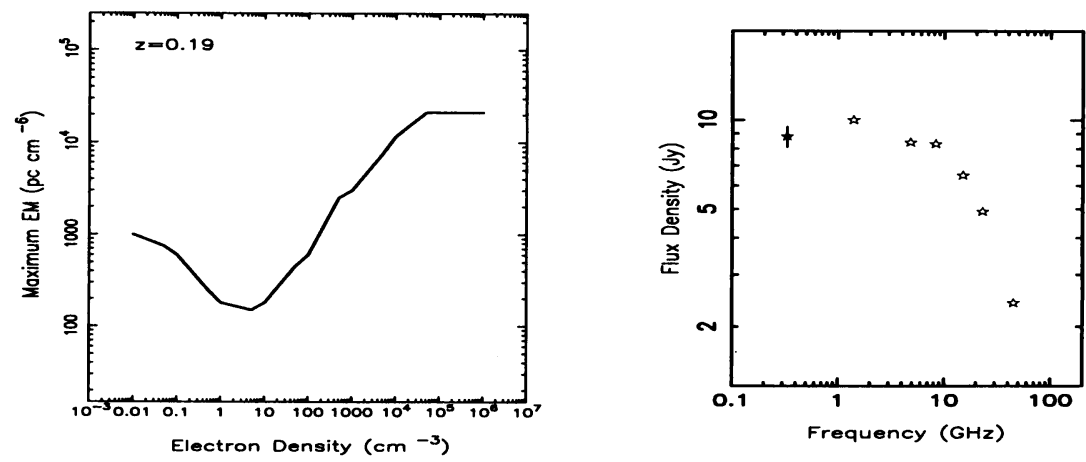

Figure 1. Left: The maximum allowable emission measure of the ionized gas in the $\mathrm{z}=0.193$ system as a function of its density, based on the upper limit to the $\mathrm{H} 158 \alpha$ recombination line. Right: The measured radio continuum spectrum of PKS1830-211

were made on 27 Oct, 1997 with $3 \mathrm{C} 286$ as the primary calibrator. Figure 1 (right) shows the continuum flux density as a function of frequency. Assuming that the core radiation at low frequencies has a flat spectrum, we derive upper limits to the free-free optical depth of $\tau_{c} \leq 0.13$ at $330 \mathrm{MHz}$. For $\mathrm{T}_{e}=7500 \mathrm{~K}$, the corresponding upper limit to the beam averaged emission measure is EM $\leq 4 \times 10^{4} \mathrm{pc} \mathrm{cm}^{-6}$ if the gas is at $\mathrm{z}=0.193$ and $\mathrm{EM} \leq 10^{5} \mathrm{pc} \mathrm{cm}^{-6}$ if the gas is at $\mathrm{z}=0.886$.

Since the HI optical depth in the $\mathrm{z}=0.193$ system is the same against both the lensed images of the quasar (Verheijen et al. 1999), we assume that the line emitting gas is uniformly distributed in a slab against the entire continuum source. Figure 1 (left) shows the maximum allowable emission measure of the gas as a function of density that is consistent with the upper limits to the line strength and the continuum optical depth, assuming a line width of $80 \mathrm{~km} \mathrm{~s}^{-1}$.

Fig 1 shows that our experiment is most sensitive to low density gas $\left(\mathrm{n}_{e}=\right.$ $1-10 \mathrm{~cm}^{-3}$ ) and indicates an upper limit to the beam averaged emission measure of $100 \mathrm{pc} \mathrm{cm}^{-6}$ for this gas in the $\mathrm{z}=0.193$ system. If the line emitting gas is predominantly of density $5-10 \mathrm{~cm}^{-3}$, then the size of such a region located anywhere in the observed beam is constrained to be less than 200-300 pc in size, assuming a homogenous gas distribution. On the other hand, if the gas is in compact structures with density $\sim 10^{3} \mathrm{~cm}^{-3}$, then its beam filling factor is constrained to be less than $6 \times 10^{-4}$. Higher resolution observations of the line at comparable sensitivities and the knowledge of the system's inclination angle will greatly improve the constraints on the ISM in the $\mathrm{z}=0.193$ absorber. The limits for the $\mathrm{z}=0.886$ system are about ten times higher.

We thank S. Nair for useful discussions.

\section{References}

Chengalur, J.N., de Bruyn, A.G., \& Narasimha, D. 1999, A\&A, 343. L79

Lovell, J.E.J. et al 1996, ApJ, 472, L5

Verheijen, M.A.W, Carilli, C., Yun, M.S., \& Menten, K. 1999, private comm. 